\title{
P283
}

\section{Finite-difference Numerical Experiments and Laboratory Testing of Attenuation and Dispersion in Patchy-saturated Media}

\author{
V. Shulakova* (CSIRO), M. Pervukhina (CSIRO), T.M. Müller (CSIRO), M. \\ Lebedev (Curtin University of Technology) \& B. Gurevich (Curtin University \\ of Technology/CSIRO)
}

\section{SUMMARY}

Accurate numerical modeling tools are of practical importance for understanding of seismic wave propagation in reservoir rocks. 2D finite-difference (FD) poroelastic code is used for simulation of acoustic wave propagation through water saturated medium with regular distributed circular gas inclusions. The results of the numerical simulations are validated with the newly derived theoretical solution for the same geometry of patches. Finally, the numerical results for a media with randomly distributed patches are used to verify the interpretation of the results of a laboratory experiment in which saturation and compressional velocity are simultaneously measured in a sandstone sample. 


\section{Introduction}

Hydrocarbon reservoir rocks usually include a mixture of fluids and gases in pores between solid grains. Patchy saturation of these rocks causes frequency-dependent attenuation and velocity dispersion that affect all parameters of the seismic wave field. Accurate numerical modeling tools are of practical importance for understanding of seismic wave propagation in reservoir rocks. Despite decades of research and a number of theoretical and numerical studies of poroelastic effects in patchy saturated media (Johnson, 2001, Wang, 2001, Masson and Pride, 2007), the numerical tools are usually not applied accurately (Krzikalla et al., 2006). In this paper we demonstrate that a poroelastic finite difference (FD) code provides us with an accurate solution for physically plausible geometries of patches and is a powerful tool for interpretation of experimental results. For these purposes acoustic wave propagation through water-saturated medium with circular gas inclusions is simulated using the 2D finitedifference (FD) poroelastic code (Krzikalla and Müller, 2007, Wenzlau and Müller, 2008). The results of the numerical simulations are validated with the newly derived theoretical solution for the same geometry of patches. Finally, the numerical results for poroelastic solids with randomly distributed patches are used to verify the interpretation of the results of a laboratory experiment in which saturation and compressional velocity are simultaneously measured in a sandstone sample (Lebedev et al., 2009).

\section{D Patchy-saturated theory}

Johnson (2001) provided exact results for attenuation and dispersion in patchy-saturated rocks for two geometries in 1-D and 3-D space: a) for periodically spaced slabs and b) for periodically spaced concentric spheres. Our numerical capabilities are confined to 2-D, where no exact solution is known to the best of our knowledge. In analogy to the concentric sphere model, we derive frequency-dependent attenuation and dispersion for a 2-D medium containing a periodic arrangement of concentric circles with inner radius $R_{1}$ (Figure 1a). According to Johnson (2001) the influence of the spatial patch distribution on acoustic signatures can be described the geometry parameter $T$ which, in this case is

$$
\begin{gathered}
T=\frac{K_{B G W} \phi^{2}}{8 \kappa R_{2}^{2}}\left(\left[\eta_{2} g_{2}^{2}+2 g_{1} g_{2}\left(\eta_{1}-\eta_{2}\right)-\eta_{1} g_{1}^{2}\right] R_{1}^{4}-2 g_{1} g_{2}\left(\eta_{1}-\eta_{2}\right) R_{1}^{2} R_{2}^{2}+\right. \\
\left.+4 \eta_{2} g_{2}\left(g_{1}-g_{2}\right) \ln \left(\frac{R_{1}}{R_{2}}\right) R_{1}^{2} R_{2}^{2}-\eta_{2} g_{2}^{2} R_{2}^{4}\right)
\end{gathered}
$$

This expression for $T$ is used together with equations 43-45 in Johnson (2001) in order to compute frequency-dependent velocity and attenuation in 2-D media. We note that the logarithmic singularity in (1) in the case $R_{1}=0$ is removable since the term is multiplied by $R_{1}^{2}$.

\section{Quasistatic poroelastic FD modeling}

We numerically simulate plane wave propagation through 2D water saturated media with circle gas inclusion (Figure 1b). Wave propagation is initiated by applying a normal stress at the top of the domain. As a loading function we use $\sin ^{3} x$, where the duration of source signal $(1 \mathrm{~s})$ and time of wave propagation ( $5 \mathrm{~s}$ with $2 * 10^{-4} \mathrm{~s}$ time step) are chosen sufficiently long to model quasistatic conditions and avoid resonance. The shape of outer water-saturated medium is a circle for analytical solution and a square for computational model. The size of model is 40 x 40 grid points. Periodical boundary conditions (PBC) are applied in lateral direction. Receiver configuration consists of 20 receiver lines with 20 receivers in each line. Distance between lines and receivers - 2 grid points. Material properties for the numerical model are the following. For rock frame - porosity -0.2 , permeability $-1^{*} 10^{-12} \mathrm{~m}^{2}$, bulk modulus $\mathrm{K}_{\text {dry }}$ $5 * 10^{9} \mathrm{~Pa}, \mathrm{~K}_{\text {grain }}-35^{*} 10^{9} \mathrm{~Pa}$, shear modulus $\mathrm{G}_{\text {dry }}-11 * 10^{9} \mathrm{~Pa}$, density $-2650 \mathrm{~kg} / \mathrm{m}^{3}$. For gas: 


\section{Amsterdam 'og}

bulk modulus $-0.1 * 10^{9} \mathrm{~Pa}$, density $-100 \mathrm{~kg} / \mathrm{m}^{3}$, viscosity $-0.1 * 10^{-5}$. For water: bulk modulus $-2.25 * 10^{9} \mathrm{~Pa}$, density $-990 \mathrm{~kg} / \mathrm{m}^{3}$, viscosity $-10^{-3}$.
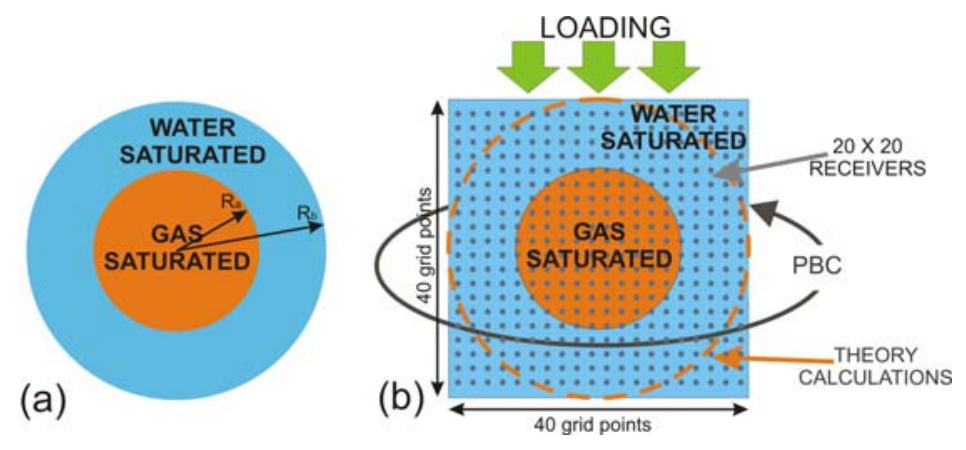

Figure 1: Schematic plot of patchy-saturated model: a $2 D$ periodic geometry with inner circle filled with gas and outer medium ( $a$ - circle for analytical solution and $b$ - square for computer model) filled with water.

As a result of numerical simulations we obtain seismograms of velocity divergence $v_{x x}+v_{y y}$, velocity difference $\mathrm{v}_{\mathrm{yy}}-\mathrm{v}_{\mathrm{xx}}$, and stresses $-\mathrm{s}_{\mathrm{xx}}-\mathrm{s}_{\mathrm{yy}}, \mathrm{s}_{\mathrm{yy}}-\mathrm{s}_{\mathrm{xx}}$. In order to obtain frequencydependent velocity and attenuation we used the method suggested by Masson and Pride (2007).

At first, integration of the bulk velocity divergence and velocity difference yields mean and differential strains. Then, stresses and strains are averaged within the model and transformed to the frequency domain by Fourier transform. Finally, effective bulk and shear moduli, fast $P$-wave velocity and attenuation are calculated from these averaged fields according to Wenzlau and Müller (2008).
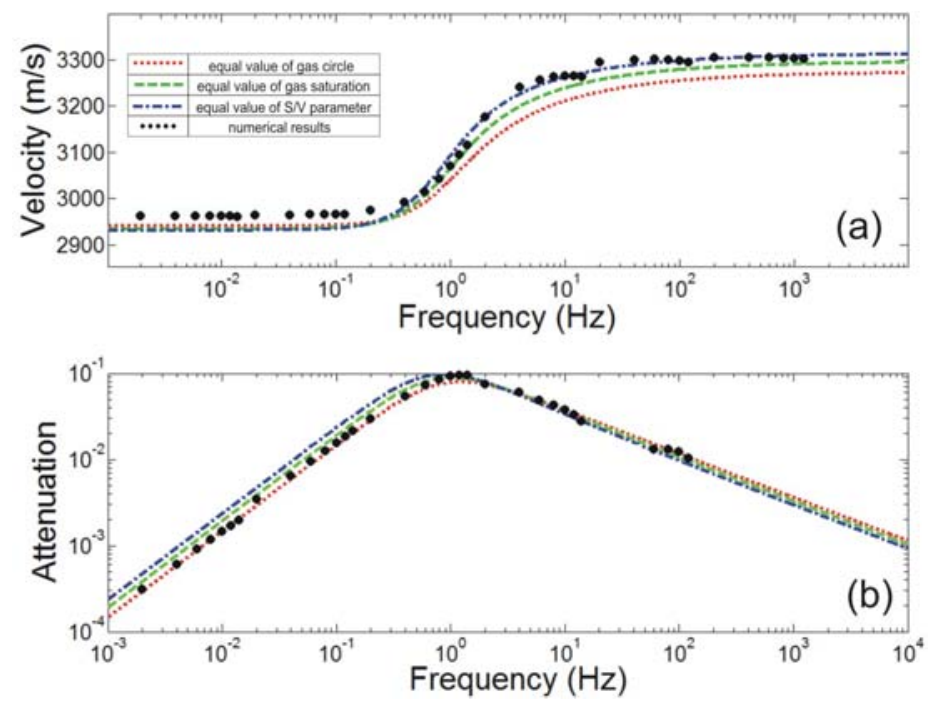

Figure 2: Three variants of theoretical prediction (dashed lines) (described in text) and numerical calculations (dots) of P-wave velocity (a) and attenuation (b) vs. frequency.

Velocity dispersion and attenuation are numerically simulated for a wide range of frequencies from $10^{-2}-10^{3} \mathrm{~Hz}$. The numerical results for water saturation of 0.75 are shown by black dots in Figure 2 in comparison with the analytical solutions for three slightly different media.

The analytical solution shown by red dotted curve is obtained for the medium with the same radius of gas inclusion as used for numerical simulations. Green dashed curve presents the analytical solution obtained for the medium with the slightly different radius of gas 


\section{Amsterdam 'og}

inclusion but the saturation equals to the saturation that used for simulations. Finally, the analytical solution for the medium for which $\mathrm{S} / \mathrm{V}$ ratio, the ratio of the boundary area between two phases to the sample volume, is equal to the $\mathrm{S} / \mathrm{V}$ ratio of the numerical model is shown by blue dot-and-dashed curve. In all cases diameter for outer circle is equal to the square side.

The analytical results for 3 different media are shown because the analytical and numerical results are obtained for similar but still slightly different models (Figure 1a, b) where the model at Figure 1a does not fill a plane.

The blue dashed-and-dotted curve obtained with the same S/V ratio fits numerical data best of all, though we still have higher values of P-wave velocities for low frequencies then those predicted by theory.

Dispersion and attenuation curves (dots) for four frequencies $(0.4,0.6,1,2 \mathrm{~Hz}$ ) versus water saturation are shown in the Figure 3 together with analytical solution calculated with the same $\mathrm{S} / \mathrm{V}$ ratio. Variations in water saturation $(0.19,0.44,0.64,0.75,0.84,0.94)$ are obtained by variation of the inner circle radius. Velocities are bounded by Biot-GassmannWood (BGW) curve for low frequencies and by Biot-Gassmann-Hill (BGH) curve for high frequencies (Johnson, 2001).
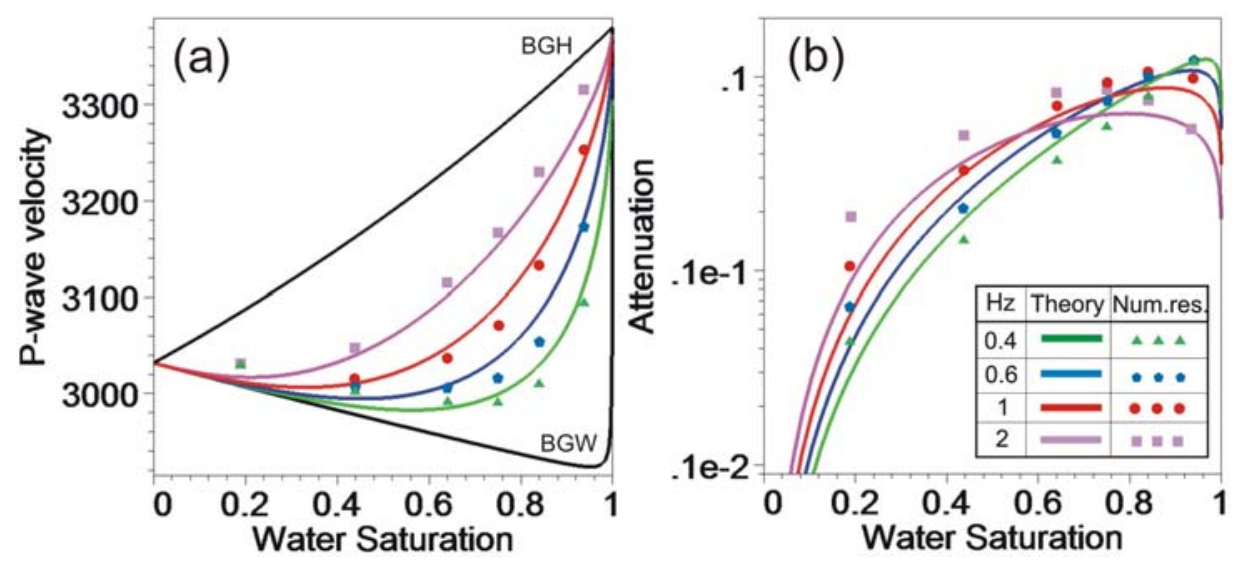

Figure 3: Theoretical prediction (solid lines) and numerical calculations (dots) of P-wave velocity (a) and attenuation (b) vs. water saturation for different frequencies.

Figures 2 and 3 show that our numerical results are in a reasonably good agreement with the theory. The slight existing discrepancies between the numerical and analytical results are most probably caused by different geometries rather than having a physical significance.

\section{Comparison with experimental data}

A numerical simulation of wave propagation in a 2-D poroelastic solid is performed to validate an interpretation of a velocity-saturation relation obtained in a laboratory experiment that described in details in Lebedev et al. (2009). Sandstone cylindrical sample is gradually saturated with water and measurements of P-wave velocity and X-ray computer tomography (CT) scans are performed simultaneously.

The velocity-saturation relation obtained in this experiment presented by black diamonds in Figure 4a shows a transition from the BGW (blue line) to the BGH (red line).

In order to numerically simulate changing degree of water saturation in sandstone sample we change the number of the randomly distributed gas patches in water saturated media. Eleven different distributions are shown in Figure $4 \mathrm{~b}$ where gas is marked as white spots on dark background which represents water-saturated media. Our numerically calculated curve presented as green circles appears to be consistent with experimental data. Numbers near circles correspond to the models from Figure $4 \mathrm{~b}$. Despite the simplified numerical setup the simulation results reproduce the overall behavior of the measured velocity-saturation relation. 


\section{Amsterdam 'og}
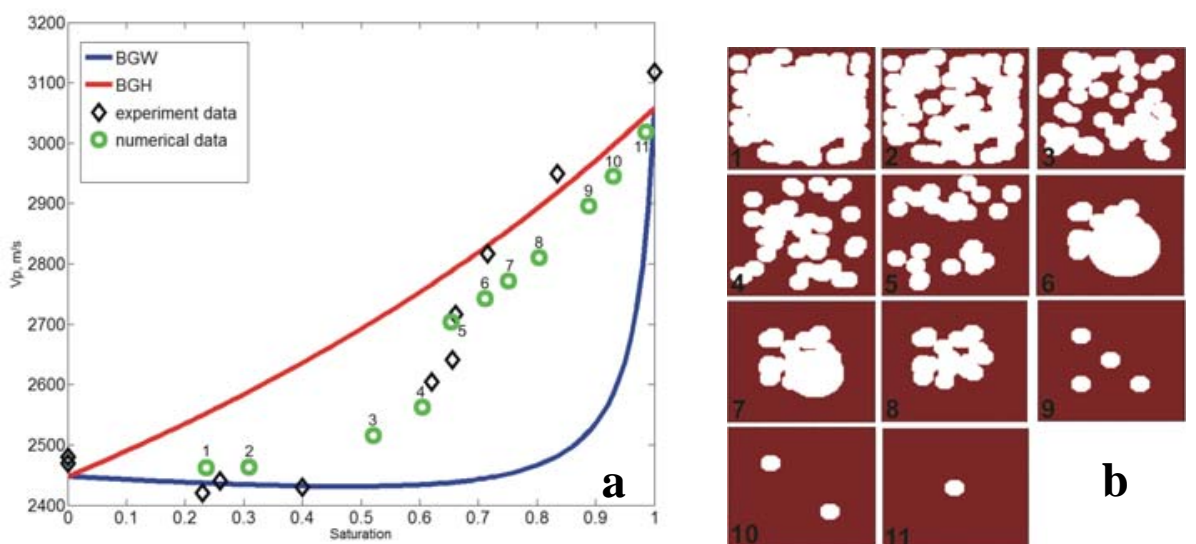

Figure 4: Velocity-saturation relation: simulation vs. experiment. (a) Comparison of experimentally measured velocities obtained from the quasi-static injection with numerical simulation of wave propagation in poroelastic media; (b) distribution of gas patches for which numerical simulations are carried out.

\section{Conclusions}

For the first time exact theoretical solution has been obtained for 2D water saturated medium with regular distributed circular gas inclusions. A good agreement of the theoretical predictions with the numerical results obtained using poroelastic FD code has been shown. Numerical simulations for the different distribution of patches have been performed to explain the results of the laboratory experiment. Simulated results reproduced the overall behaviour of the measured velocity-saturation relationships. The presented promising results may serve as a starting point for simulation of wave propagation in porous media with more complex fluid patch patterns.

\section{References}

Johnson, D.L. 2001. Theory of frequency-dependent acoustics in patchy-saturated porous media, J. Acoust. Soc. Am., 110: 682-694.

Wang, X. 2001. Seismic wave modelling in poroelastic media using a high-order finitedifference method, SEG Extended Abstracts, SM1.1.

Masson, Y.J., Pride, S.R. 2007. Poroelastic finite-difference modelling of seismic attenuation and dispersion due to mesoscopic-scale heterogeneity, Journal of Geophysical Research, 112.

Krzikalla, F., B. Hardy, T.M. Müller, and B. Gurevich, 2006, Seismic wave attenuation and dispersion in patchy-saturated rocks: Numerical experiments: $68^{\text {th }}$ EAGE Conference, Vienna, Extended Abstracts, E038.

Krzikalla, F., Müller, T.M. 2007. High-contrast finite-differences modeling in heterogeneous poroelastic media, SEG Expanded Abstracts, 26: 2030-2034.

Wenzlau, F., Müller, T.M. October 2008. Finite-difference modeling of wave propagation and diffusion in poroelastic media, Geophysics (revised version submitted).

Lebedev, M., Gurevich B., Clennell B., Pervukhina M., Shulakova V., Muller T.M. 2009. Laboratory observation of velocity-saturation relation transition during water imbibition of porous rock, Biot Conference Extended Abstract. 